\title{
@
}

\section{Dire le lieu pour faire naître des histoires : Claudie Hunzinger, de Bambois à La Survivance}

\author{
Pierre Schoentjes \\ Ghent University
}

\section{Propriété(s) du lieu-dit}

À l'été 1964, Claudie Hunzinger et son mari, Francis, partent s'installer à la Bourse Noire, lieudit situé sur le territoire de Fréland dans les Vosges, avec l'intention d'y élever des moutons. Ils quitteront les lieux en janvier 1966 pour continuer la réalisation de leur projet à Bambois, sur la commune de Lapoutroie ${ }^{1}$.

Comme beaucoup de jeunes de leur génération, ils rejetaient la société de consommation et espéraient trouver dans la vie rurale une existence plus conforme à leurs idéaux. « Nous étions dans les fleurs jusqu'au cou et les baisers $»^{2}$ écrit Hunzinger dans Bambois ou la vie verte, qui relate neuf années de vie au fil desquelles l'artisanat du tissage est venu compléter l'activité d'élevage. Elle concède aussi : « Nous étions vraiment perdus à cette époque, je veux dire que nous ne nous étions pas encore trouvés nous-mêmes, ni quoi que ce soit » $(V V: 13)$. Jean-Claude Barreau, préfacier de ce témoignage prenant place dans une collection qui explore des modes de vies alternatifs, écrivait : "C'est que notre société trépidante et polluante en même temps qu'elle exacerbe les besoins de silence, de communion, de vie naturelle, si gravement insatisfaits, permet par son abondance même la résurgence de modes de vie considérés jadis comme dépassés et qui sont en fait d'avenir » $(V V: 13)$. L'époque est à l'optimisme, d'autant plus partagé que le retour à la nature s'est à cette époque délesté en grande partie de la charge réactionnaire qui l'avait longtemps rendu suspect.

Quarante ans plus tard, Hunzinger, devenue écrivaine sans pour autant avoir abandonné son activité de plasticienne, publie chez Grasset La Survivance. Ce roman prend pour sujet l'installation de deux libraires, ruinés par la crise du livre, dans une maison qui donne son nom au roman. Il s'agit d'une vieille masure où ils vont vivre une existence de pionniers. Malgré la grande précarité de leur installation, et la rudesse des travaux qu'ils doivent accomplir pour survivre, le couple trouve un

\footnotetext{
${ }^{1}$ Ce texte constitue une première version d'une étude qui a été complétée depuis, suite à la parution de deux autres ouvrages de Hunzinger. Il a donné lieu à une version remaniée - "L'Architecture des branches 》- dans Les Moments littéraires $\left(\mathrm{n}^{\circ} 42\right.$, 2019, p. 7-31). Le texte définitif trouvera sa place dans un volume intitulé. Littérature et écologie. Le mur des abeilles, à paraitre en 2021 chez José Corti.

${ }^{2}$ HunZinger Claudie, Bambois, la vie verte, Paris, Stock2, coll. «Vivre », 1973, p. 17 ; dorénavant VV.
} 
équilibre et même une forme de bonheur grâce au souvenir des livres qui ont nourri leur imaginaire et qui donne désormais sens à leur vie.

Dans l'intervalle, le récit de 1973 avait été réédité en 1979 : l'éditeur Stock l'a toutefois fait passer de sa collection "Vivre » à une collection " Nature ", et cette migration invite à modifier la perspective de lecture. Ce qui se présentait comme le témoignage d'une expérience sociale - l'édition originale comportait ainsi la reproduction de documents authentiques, comme le courrier de la S.A.F.E.R. annonçant l'octroi du prêt pour l'achat de la ferme - devient sous la nouvelle couverture un récit qui s'inscrit dans le cadre de l'écriture de la nature ${ }^{3}$. Le genre connaît une popularité croissante à la fin du XX ${ }^{\mathrm{e}}$ siècle, portée par la nature writing américaine qu'une maison d'édition comme Gallmeister s'attache à faire connaître. Ce changement d'orientation est d'autant plus manifeste que le titre a été modifié : Bambois, la vie verte se trouve réduit à Bambois. L'œuvre s'inscrit ainsi dans le paradigme du célèbre Walden de Henry D. Thoreau, fondateur du récit de la solitude au sein de la nature et qui prenait déjà pour titre le nom du lieu-dit où il s'était installé.

Jean-Pierre Martin, écrivain et universitaire qui a participé au mouvement de «retour à la nature »-qui était en réalité bien plus un aller vers la nature dans la mesure où la plupart de ceux qui tentaient l'aventure n'en avaient aucune expérience -, est revenu sur cette période dans Sabots suédois. Au sujet de la Claudie Hunzinger de Bambois, il observe : « Elle y formulait le désir de beaucoup d'entre nous $»^{4}$. La volonté d'entamer une vie nouvelle dans un cadre sauvage voire primitif, pousse en effet à l'époque toute une jeunesse à partir en quête de lieux naturels propices. Des lieux-dits, en d'autres termes, et que Martin caractérise ainsi :

Lieu-dit : de «lieu» et de « dit». Lieu de campagne qui porte un nom traditionnel désignant une particularité d'ordre topographique ou historique. Lieu qui n'existe que parce que, parfois, on le dit, et dans cette seule mesure. Il peut nommer un vallon, un bout de désert. Il affirme le plus souvent la prééminence du cours d'eau, du vent, du bosquet, sur l'échafaudage. Je me suis donc mis, comme bien d'autres, à chercher un lieu-dit sur la carte de la France. Ce n'était pas ce qui manquait. $(S S: 20)$

Non sans humour, Martin pointe le fait que le lieu-dit n'existe que dans la mesure où son nom est (rarement) prononcé... Mais en écrivant Bambois, Hunzinger va précisément faire exister le lieu audelà de l'oralité. La Survivance, dont le projet n'est plus de l'ordre du témoignage mais du littéraire, ira plus loin encore en inscrivant le lieu de solitude dans un imaginaire partagé. D'une œuvre à l'autre, l'on observe que le lieu passe d'un statut de propriété personnelle à celui d'espace partagé, comme l'est tout lieu qui existe d'abord par la fiction.

La question de l'acquisition des fermes et de l'obtention des crédits nécessaires pour s'établir occupait une large place dans Bambois. Posséder une maison compte, sinon pour Francis, du moins pour Claudie : «J'aime l'idée d'une maison, d'un point de repère ancré dans la terre » $(B, \mathrm{p} .16)$. La manière dont l'acquisition de la première ferme est dramatisée illustre cette importance, la tension montant parce que le précédent propriétaire s'était engagé à la vendre à deux acheteurs. C'est finalement admonesté par le notaire que l'homme, profondément troublé et incapable de parler, « désigne du doigt » (VV, p. 19) ceux à qui il destine sa ferme.

Le couple est si heureux de s'installer dans un lieu qui leur parait idéal qu'ils ne prennent pas garde aux menaces contenues dans le toponyme :

Un jour, nous apprenons que Bourse Noire est une appellation qui viendrait de bosse nord, qu'elle est placée entre un pré dit "peines perdues » et un autre nommé « la bâche du loup ». Nous sommes si insouciants que nous ne nous préoccupons guère de ces signes noirs du destin, nous prévenant que notre nid est en plein nord, et que les foins y seront maigres et durs à récolter. $(V V$, p. 16)

Le positionnement de La Survivance par rapport à la question du lieu se présente différemment. D'abord parce que malgré son statut de roman, le texte entend situer géographiquement le lieu avec précision : ce calque de Bourse Noire et de Bambois est situé dans le massif du Brézouard, entouré d'un

\footnotetext{
${ }^{3}$ Lire à ce sujet notre étude SchoentJes Pierre, Ce qui a lieu. Essais d'écopoétique, Marseille, Wildproject, 2015, p. 86, 87 ; p. 108-112.

${ }^{4}$ MARTIN Jean-Pierre, Sabots suédois, Paris, Fayard, 2004, p. 32 ; dorénavant SS.
} 


\section{DIRE LE LIEU POUR FAIRE NAÎTRE DES HISTOIRES}

paysage que le texte rend visible. Ensuite parce qu'il s'agit bien cette fois pour les vieux libraires d'un retour à la nature puisque l'endroit est celui où, jeunes, ils avaient déjà tenté de s'installer. L'expérience s'était toutefois soldée par un échec. Mais il se trouve surtout que pour Sils et Jenny, la question de la propriété ne se pose plus comme pour Melu(sine), - c'est-à-dire Claudie - et Pagel - alias Francis -, les pionniers de Bambois: «La Survivance était une maison qui nous appartenait encore parce que impossible à vendre $»^{5}$. La possession matérielle est passée au second plan. L'installation ne se fait d'ailleurs plus dans un endroit chargé de tous les rêves de la jeunesse, mais dans un lieu au contraire dépouillé de toute idéalisation. Le texte n'en réclamant pas la propriété, le lecteur peut aisément se l'approprier.

\section{Beauté du lieu}

Bambois était porté par un lyrisme et une fougue que les critiques de l'époque n'ont pas manqué de relever, pointant le "forcing de l'enthousiasme à base d'attendrissement et d'indignations " ${ }^{6}$ même quand leur jugement global du livre était favorable. Si Pagel note sobrement « Je suis heureux » $(B$, p. 32), Melu marque volontiers son émerveillement devant le monde. Ici devant une Angélique archangélique qu'elle n'identifie pas de prime abord: "C'est assez magique à voir ; un feuillage gigantesque d'où s'est élevé une tige, une vraie colonne de temple grec, cannelée et portant à son extrémité un bouton floral de la taille d'un œuf d'autruche » $(B$, p. 90$)$. La beauté et l'enchantement sont dits de façon explicite. Le récit publié dans les années 70 rejoint par là une certaine esthétique de l'époque, qui ne craignait pas d'insister sur la fusion avec les éléments :

A Bambois, je suis Bambois. Je suis paysage. J'ai mille-feuilles, mille-pattes, mille-pertuis, mille-yeux, mille doigts, et une immense peau qui n'en finit pas, de l'est à l'ouest, du matin au soir, de la naissance à la mort. Ici, c'est la paix. Rien ne se jette sur moi [...] Je sens tout. Je suis bien. Je suis tout. Je suis. ( $B$, p. 220, 221)

La dimension lyrique du récit de Hunzinger s'observe encore quand elle rapproche Paris et Bambois en mai 68 :

Les usines, les lycées sont fermés. Paris explose. Ici la révolution de mai, c'est l'explosion du printemps. Je m'installe à l'ombre d'un pommier, et je retrouve les saveurs des étés adolescents : cela vient de cette impression de vacances, de mes pieds nus et aussi de l'activité joyeuse que m'apporte le rouet. (B, p. 118)

Les « gauchistes », dont les plus visibles se tenaient à l'époque éloignés de la campagne et plus encore de l'écologie, ne goûtaient pas nécessairement ce genre de prose trop peu radicale à leur goût, et certainement pas ouvriériste. Lors de la parution de 1973, Charlie Hebdo raillera le «style MarieAntoinette » de Bambois en faisant mine de craindre que « la dernière pollution ne soit la littérature de gardien de moutons $»^{7}$. Le journal satirique reviendra à la charge quelques numéros plus tard pour reprocher à Hunzinger que ses «joies à être proche d'une nature préservée » ressemblent à celles d'un maharadja « qui décrirait pour son bon peuple de chameliers faméliques, ses joies dans son palais doré ». Et d'apostropher l'écrivaine :

Mais qu'est-ce qui se passerait si tous les gogos que votre charmant petit livre va faire rêver se précipitaient à leur tour pour envahir le voisinage ? Ça serait vite de nouveau le merdier. Vous savez bien que ce n'est pas la solution. C'est seulement votre solution, anecdotique et pittoresque, rien de plus ${ }^{8}$.

\footnotetext{
${ }^{5}$ HunZINGer Claudie, La survivance, Paris, Grasset, 2012, p. 25 ; dorénavant $L S$.

${ }^{6}$ Soudet Pierre, « Les moutons des Vosges », L'Express, 21-27 mai 1973, p. 157.

${ }^{7}$ De Ton Delfeil, pseudonyme de Roussel Henri, «Bambois, la vie verte, bouquin de Cl. Hunzinger », Charlie Hebdo, $\mathrm{n}^{\circ} 124$, lundi 2 avril 1973, p. 13.

${ }^{8}$ Isabelle, « Bambois, La vie verte », Charlie Hebdo, n 129, lundi 9 mai 1973, sp.
} 
Dans le roman de 2012, l'enthousiasme bucolique est largement tempéré et la joie se dit de manière plus retenue :

La Survivance est creusée dans la pente de la montagne comme une halte minuscule où nous avons décidé de nous arrêter. L'enchantement vient de la somptuosité, du luxe de l'été, et des fenêtres ouvertes, des pieds nus, des habits légers. Il fait bon. ( $L S$, p. 51$)$

Le lieu est désormais désigné par le nom de la maison, qui se fait lieu-dit. Le sens ne se définit toutefois plus à travers les connotations de l'étymologie mais par le biais de souvenirs de lectures, à commencer par celle du grand historien de l'art Warburg qui nommait survivance «la capacité des images à revenir nous hanter » $(L S$, p. 162). Alors que pour la Bourse noire les pionniers héritaient d'un lieu dont la signification - en lien avec la difficulté du travail de la terre - préexistait à leur venue, ce sont maintenant les exilés qui à travers le nom donnent sens à l'ancienne maison de mine qu'ils vont habiter. Mais le jeu n'est pas qu'intellectuel et ils sont parfaitement conscients que le mot " survivance » renvoie aussi très concrètement à la difficulté à s'organiser dans la solitude d'une nature rude : " Il nous arrivait de penser que nous n'allions survivre ni à la montagne, ni à la décomposition globale » $(L S$, p. 90).

\section{Instantanés : fixer le lieu}

Bambois incline vers le lyrisme et insiste sur la fusion avec le lieu. Y participent des métaphores, dont certaines, inspirées par l'activité pastorale, reviennent régulièrement : "mille et un moutons passent, poussés par le vent $\gg(B$, p. 35). L'image sera répétée dans un paragraphe qui s'arrête sur la mère de Pagel, qui avait participé au mouvement du Contadour. Le premier voyage du couple avec la nouvelle $2 \mathrm{CV}$ consiste donc en un pèlerinage sur les lieux de Giono, à la recherche de moutons : " Nos premiers troupeaux sont ceux des nuages, infinis et moutonnant $\gg(B$, p. 45). Mais déjà dans les années 70 Hunzinger possédait le sens de la petite notation précise, qui peut faire penser à un Jules Renard. Elle observe un lérot tombé dans une carafe de lait: "Il s'était tellement démené qu'il en avait fait du beurre » $(B: 95)$. Certaines de ces petites scènes tirées de l'expérience directe voyageront du récit au roman. Celle-ci notamment, qui témoigne des lectures de jeunesse : «Francis Ponge a été oublié sous l'averse : surpris, un orvet s'y est abrité » ( $B$, p. $22 ; L S$, p. 51).

La Survivance dépassera toutefois l'anecdote, fût-elle significative, pour donner une profondeur supplémentaire au réel en établissant les rapports essentiels que la vie entretient avec les livres, et audelà avec tout un savoir culturel ${ }^{9}$. Sans lourdeur, et même avec humour, comme quand l'ânesse se met un jour à mâcher un volume De la nature des choses, littéralement donc " manger le livre » comme dans la parabole d'Ezéchiel. L'écrivaine commente : «Ce qu'elle cherchait à capter, maintenant, je le sais. C'était, avec l'angoisse de notre fin, le contraire de l'angoisse; le calme de la raison, la saveur de Lucrèce » $(L S$, p. 204).

Les métaphores du roman se font plus originales ; elles s'ancrent dans le concret tout en invitant aux associations :

Il tombait du grésil, ou plutôt du mimosa, des grains pâles poudrés de froid, c'était ravissant et très inquiétant, car je ne pouvais m'empêcher de penser à La Ballade de Narayama et que nous nous livrions à la nature, au mauvais temps, pour notre dernier voyage. ( $L S$, p. 54$)$

L'écriture donne forme au lieu, les lectures transforment la vie. L'existence n'est plus seulement quotidienne, elle devient unique parce qu'elle s'enrichit de souvenirs des livres, des tableaux, des films. Là où Bambois, qui opte pour une forme qui rappelle celle du journal voire d'un album de photos, montrait les lieux dans leur immédiateté matérielle, La Survivance les saisit dans une durée qui est à la fois celle des saisons qui passent et celle des lectures qui s'accumulent :

\footnotetext{
${ }^{9}$ Pour une analyse détaillée de cette pratique, essentielle chez Hunzinger, on lira : ScHOENTJES Pierre, « Solitaires dans la nature :

"L'art est ce qui rend la vie plus intéressante que l'art" ", Les Temps modernes, n695, 2017, p. 155 sv.
} 


\section{DIRE LE LIEU POUR FAIRE NAÎTRE DES HISTOIRES}

Le lundi de Pâques, nous avons fini d'emballer. Le mardi, nous sommes partis par de petites routes irradiées de cerisiers en fleur, sous de gros cumulus neigeux, escortés au loin par des sommets blancs qui étincelaient comme des icebergs, des mondes séparés de notre monde, des mondes en soi. ( $L S$, p. 30$)$

Les lieux ne sont pas définitivement figés, ils sont habités par une réalité dont le lecteur prend conscience à travers une série de dévoilements progressifs :

L'air de la montagne était inquiété par des craquements. Là-bas, sous les sapins, une bête prise d'un vague à l'âme insurmontable lançait des appels, d'incessants petits cris fous, plaintifs. Quelque chose d'autre guettait. (LS, p. 250)

Des loups... réels sans doute, mais qui, à l'extrême fin du roman d'où ces lignes sont tirées, constitueraient aussi une image de cette mort auquel le couple cherche à donner une place qui ne recouvrirait pas les joies de la vie dont ils gardent la mémoire.

Avec le recul, le mouvement vers la nature des années 70 pouvait passer, aux yeux mêmes de ceux qui avaient tenté l'expérience, comme une fuite. Par contre, l'installation en forêt à un âge avancé exprime une volonté de faire face. La narratrice de 2012, qui a retenu la leçon de Vacances de Ray Bradbury, a pleinement conscience qu'en Arcadie « à la longue, [on] s'emmerde » (LS, p. 139). C'est précisément pour continuer à vivre dans l'action et de manière pleinement consciente en attendant la mort que le couple s'installe en forêt, et dans la solitude.

\section{La solitude de l'ermitage dans la sauvagerie}

Bambois évoquait déjà, en demi-teinte, la question de la solitude : «Il arrive, le soir, que nous nous découvrions seuls, vraiment seuls, tous les deux. Nous sommes seuls à Bambois, isolés en montagne, et dans notre façon de vivre » $(B, \mathrm{p} .107)$. Le récit thématisait par ailleurs la question de l'isolement choisi en relatant la rencontre avec un ermite, celui-là même qui apprendra au couple à tisser. Pour autant, les relations humaines - avec l'administration, les amis de passage, les clients qui leur achètent bêtes et pièces de laine, leurs enfants qui grandissent - occupent une place extrêmement importante dans le récit. Melu se déplace pour assurer des cours à Colmar, Pagel voyage dans le cadre de ses activités comme berger : Bambois est le récit du social.

La Survivance, en contraste, est le roman d'une solitude assumée jusque dans ce qu'elle peut avoir de moins attrayant, comme la déchéance des corps. Entouré d'animaux, vivant dans un environnement qui leur appartient plus qu'à l'homme, le couple a conscience de son isolement : «On habitait une sorte d'île ou de minuscule Cité interdite. Une zone à part» ( $L S$, p. 117). De manière caractéristique, le texte multipliera les images de l'île. Les baies de genévrier qu'ils consomment quand la disette frappe, possèdent malgré les circonstances « un goût d'île découverte » (LS, p. 186). Il est toutefois bien entendu qu'ils vivent sur une « île en montagne » $(L S$, p. 82$)$, pas dans une île ensoleillée où la vie serait facile parce que la nature s'y montre accueillante et prodigue.

Le vieux couple se prépare à sa vie en autarcie dans la forêt en faisant des préparatifs pratiques, rassemblant outils, matériel de camping, mobilier de base; ils prennent soin aussi de se munir de " plusieurs cartes IGN» $(L S$, p. 43) afin d'explorer les lieux. Mais la nécessité de subvenir à leurs besoins les ramène aussi vers les livres. Dans la mesure où un potager sera indispensable à leur survie, Jenny lit Vassily Peskov, qui a retracé la vie d'une famille de vieux-croyants retranchée du monde à partir de la fin des années 30 : «J'avais relu Ermites dans la Tä̈ga, réfléchissant beaucoup, prenant des notes » $(L S$, p. 66). S'ils ont quitté leur librairie par nécessité, c'est par choix qu'ils optent pour une vie de reclus.

Ils ne partent pas avec l'espoir d'une vie meilleure, mais pour éprouver sa densité au quotidien. De ce point de vue, le nom qu'ils donnent à leur ânesse est significatif : dans Bambois celle-ci se nommait Utopie, l'animal qui les accompagne dans La Survivance porte le nom d'Avanie... C'est une vie de dépouillement qu'ils choisissent de vivre : « J'ai préféré rester m'occuper du jardin et des petites poules et d'Avanie. Être seule. Expérimenter le sens du mot anachorète. M'habituer à ne pas avoir peur » (LS, p. 133). 
Le texte multiplie les allusions aux ermites et aux anachorètes. Ils entendent aller même plus loin que certains reclus anciens qui vivaient de la charité et maintenaient donc un contact minimum avec la communauté. Leur isolement n'est pas aussi extrême que celui de Christopher T. Knight qui a récemment vécu 27 ans dans l'isolement complet autour de Pound Lake dans le Maine ${ }^{10}$, mais il est bien réel. À la différence toutefois de Knight, Sils et Jenny entendent vivre à deux et pas seuls, et entourés de la forêt et de ses habitants, les animaux. Sans vivre caché, le couple sait se faire oublier : "Personne ne sait que nous sommes là » $(L S$, p. 51$)$ et plus loin « Nous aussi, nous étions discrets. Personne ne savait que nous habitions ici. Ni les bêtes ni les humains » $(L S$, p. 88$)$.

Il s'agit certes de passer inaperçus aux yeux des Brigades vertes qui pourraient trouver à redire à leur installation dans une maison légalement inhabitable mais aussi d'échapper aux regards parce que des intrusions extérieures trop fréquentes mettraient en péril le mode de vie qu'ils ont choisi. Bambois s'arrêtait déjà à cette problématique en observant que lorsque leur petite notoriété s'était s'agrandie en raison du succès que connaissent les tissages, les visites d'inconnus, en augmentation constante, avaient de plus en plus été vécues comme des intrusions ( $B$, p. 182).

La solitude est ce qui permet de retrouver du temps pour le couple, elle libère aussi du temps pour prendre conscience du monde naturel. C'est ce que rappellent quelques lignes en contradiction apparente avec l'insistance de l'auteur sur leur invisibilité dans le paysage : «j'avais vite remarqué quelque chose de bizarre. Nous n'étions pas seuls, là-haut, comme je le croyais. Il y avait du monde. Ils étaient nombreux. La nuit, ils venaient » $(L S, \mathrm{p}$. 75$)$. Inexistants, les ermites ne le sont donc qu'aux yeux des hommes, les animaux ont depuis longtemps noté leur présence.

\section{Animaux domestiques et bêtes sauvages}

Le rapport aux animaux change de manière fondamentale entre Bambois et La Survivance. Le récit des années 70 rappelle que Pagel a étudié à la Bergerie nationale de Rambouillet pour se préparer à son métier : le projet d'élevage que le couple met en place est central. C'est en fonction de cette activité qu'ils lisent le paysage : quelles sont les surfaces qui permettront de faire du foin, où les moutons pourront-ils pâturer? Le regard est d'abord utilitaire : " être éleveur n'est pas un sport du dimanche » $(B$, p. 32). L'attention se concentre donc logiquement sur les animaux domestiques et d'élevage : choix de la race de moutons, description de l'agnelage, problèmes de fourrage, menace des maladies, etc. Dans la mesure où ils élèvent aussi des moutons pour la viande, la question de l'abattage se pose :

Voilà que l'histoire est moins drôle, que ce n'est plus du tout joli, comique et attendrissant. Nous avons été pris : les agneaux, il faut les vendre. Il faut les engraisser, pas pour la vie et les pâturages et ses ruisselets, non, pour les bouchers. Pire, pour la Coopérative des bouchers. Ici, en Alsace, c'est comme ça pas de S.I.C.A. mais une Coopérative des bouchers. Tous les ruisselets de sang réunis en une crapuleuse, énorme mare. Ah, le sale moment. (B, p. 96)

La sensibilité qui s'exprime ici n'est à l'évidence pas celle de campagnards qui auraient vécu depuis toujours de l'élevage des bêtes, mais d'anciens citadins qui découvrent les réalités que beaucoup trouvent plus commodes d'ignorer.

Pour mener à bien son entreprise d'élevage, le couple s'est largement documenté et Bambois mêle à chaque page savoir livresque et récit d'expérience personnelle. Ce même aller-retour entre les livres et la vie s'observe quand ils complètent leur activité principale par l'artisanat. Le récit de la cueillette des meilleurs ingrédients naturels qui permettent de teindre la laine - plantes, racines, lichens, etc. - se développe dans de nombreux paragraphes et invite le lecteur à penser que la nature est le lieu qui pourvoit aux besoins des hommes. L'environnement naturel est rarement considéré pour lui-même et même des expériences apparemment gratuites, comme cette rencontre avec ce " sphinx tête-de-mort [qui] a heurté le carreau comme un voyageur égaré, vêtu de velours jaune » $(B$, p. 29), se justifient d'abord en raison du plaisir esthétique qu'elles procurent et des métaphores qu'elles permettent de tisser.

\footnotetext{
${ }^{10}$ Sur ce sujet, qui rejoint l'expérience de Christopher McCandless popularisée par le livre de Jon Krakauer Into the Wild, on lira : Finkel Michael, The Stranger in the Woods, New York, Penguin Random House, 2017.
} 


\section{DIRE LE LIEU POUR FAIRE NAÎTRE DES HISTOIRES}

Les animaux sauvages sont regardés à partir du point de vue des éleveurs, sans toutefois les préjugés habituels des ruraux. Le couple reconnaît l'utilité de " nuisibles » comme les renards, dans la mesure où ils se chargent de faire disparaître les cadavres des moutons : " les purificateurs. Les charognes sont sucre et aubaine pour eux » $(B, \mathrm{p} .60)$. Les animaux de la forêt font cependant partie d'un décor éloigné, ils n'appartiennent pas au même lieu, mais font intrusion : «Des hardes de chevreuils autour des pommiers à cause des dernières pommes rouges. Tant de traces que la neige a fondu » $(B$, p. 123).

La perspective change de manière radicale avec La Survivance où l'attention se déplace de l'espace domestique aux lieux de la sauvagerie. Certes, l'espace continue à être pensé initialement aussi en fonction de certains animaux qui accompagnent les pionniers âgés, en particulier l'ânesse et la chienne. La narratrice tente ainsi de convaincre son compagnon au départ en argumentant : « Et là-haut nous aurons un pré pour Avanie. Pense à Avanie. Pense à Betty » (LS, p. 26). La masure sera d'ailleurs aménagée à la manière habituelle des vieilles fermes ; l'espace est partagé : « une porte unique ouvr[e] de part et d'autre sur le monde des humains et sur celui des bêtes » (LS, p. 71). Mais même la chienne n'est plus comme dans Bambois l'aide du berger ou la compagne de jeu de la fille du couple, c'est maintenant un être à part entière dont Jenny s'efforce de comprendre le monde : " j'ai raconté à Sils comment j'avais observé le point de vue d'un chien. Il m'a dit tu as raison, étudie-le, c'est le moment ou jamais. » $(L S$, p. 16$)$.

La proximité avec les animaux domestiques permet d'imaginer plus facilement le monde à travers leurs yeux, à l'opposé les bêtes sauvages apparaissent plus étrangères. C'est sur elles pourtant que La Survivance va se concentrer et sur une nature qui n'a pas été aménagée par l'homme. La narratrice surprend « des fragments de sauvagerie, par éclairs » :

ce renard foncé, à l'étrange queue touffue mais tronquée court, coupée sans doute par un coup de fusil, et qui savait très bien où il filait, avec un air méchant et averti (quelque chose qui pouvait ressembler à de la haine), mais qui ne m'avait pas vue. Le lendemain, faisant un tour de reconnaissance dans les moraines, un autre renard s'est échappé sous mes yeux, fauve, intact, long, magnifique, sans doute la femelle de la sombre canaille de la veille. ( $L S$, p. 87$)$

L'anthropomorphisation n'est pas ici au service d'un jugement moral comme l'on pouvait en trouver - sur le renard, précisément - chez un Buffon ; c'est au contraire une manière de rapprocher les univers des hommes et des bêtes. Les rencontres avec les animaux sont des moments privilégiés, comme ce contact au petit matin avec une buse :

Elle m'observait tantôt par la droite, tantôt par la gauche, et sa petite tête pivotait pour percer le sens de mon immobilité. Elle était proche, bec et serres jaune d'or, poitrail blanc, queue trempée dans l'encre. Moi aussi j'étais proche, allongée sur le rocher. ( $L S$, p. 135).

L'observatrice est aussi l'observée et Jenny a conscience qu'elle n'est qu'une invitée dans un univers qui appartient d'abord aux bêtes sauvages. Les territoires initialement séparés du couple et des bêtes de la forêt finissent cependant par s'interpénétrer. Les cerfs, longtemps invisibles, se montrent une première fois sur des lieux qui sont tellement les leurs que le texte les désigne du terme d' « aborigènes ", comme pour mieux souligner leurs droits de premiers occupants :

L'obscurité allait tomber, quand j'ai vu sortir de la forêt une autre forêt menaçante : une troupe de cerfs aux larges ramures qui d'un bloc m'a fait face avant de se remettre à lentement progresser vers moi. Je me suis avancée à mon tour. La troupe s'est arrêtée et m'a refait face. $(L S$, p. 93)

Une nouvelle fois, le regard est essentiel et il va de l'homme à l'animal aussi bien que de l'animal à l'homme. L'image de la forêt de bois de cerfs sortant de la forêt d'arbres souligne qu'Hunzinger entend placer la nature au centre du texte. Alors que les deux pionniers avaient pensé s'installer dans un lieu qui leur appartenait, ils prennent rapidement conscience qu'il n'en est rien, entourés qu'ils sont de zones de remise et de gagnages : "Les cerfs avaient ainsi tout loisir d'aller se nourrir sur l'une ou l'autre prairie, ce qui pour eux était un avantage indéniable. Et surtout, aucun chemin 
communal ne traversait notre territoire, ou plutôt le leur » (LS, p. 117). Alors que Bambois ne prenait pas en compte le fait que les lieux appartenaient d'abord aux bêtes sauvages, La Survivance va se concentrer précisément sur cette donnée, conformément à notre sensibilité de $\mathrm{XXI}^{\mathrm{e}}$ siècle qui accorde aux animaux une place plus importante que naguère.

\section{Frontières et territoires}

Dans la mesure où Jenny s'inscrit dans les lieux non seulement par le travail manuel nécessaire à l'installation et à la survie, mais aussi par de très nombreuses lectures, elle va approfondir la question de la sociabilité des grands cerfs à travers l'éthologie et découvrir ainsi « des documents vécus, comme Le Clan des cerfs de Jean-Pierre Verhoeven » (LS, p. 96). Les livres complètent l'observation personnelle et donnent un sens à des petits faits qui, pris de façon isolée, n'en auraient aucun. Le texte rejoint ainsi cette interrogation contemporaine majeure qui porte sur ce que les animaux ressentent et la manière dont ils pensent : "Si un cerf, vivant en duo avec un autre cerf, vient à perdre son ami, l'année suivante, le survivant développe une ramure irrégulière, bizarre, mal fichue. De chagrin. Les bêtes connaissent le chagrin » (LS, p. 156).

C'est l'empathie envers l'animal qui explique la proximité avec un infirmier de rencontre qui profite de ses loisirs pour faire de la photographie animalière et, en contraste, la condamnation des chasseurs. Cette détestation remonte d'ailleurs à Bambois qui voyait déjà Melu invectiver un de ces industriels gueulards, « ridicule avec sa casquette à pompon » $(B$, p. 160). Un jour que Jenny pénètre dans une cabane, elle découvrira des photographies de scènes de chasse, illustration de la " longue histoire des prédations, toujours la même », perpétrée par des « hommes vêtus comme des légionnaires » ( $L S$, p. 226). Mais la narratrice ne s'érige pas pour autant systématiquement en juge. La compréhension plus approfondie du monde animal n'aboutit jamais à formuler des réponses toutes faites, elle sert une interrogation complexe :

Et où était la différence entre le fait d'abattre des bêtes sauvages et les adorables petites vaches vosgiennes qui pâturaient plus bas ? Car il y avait une différence. De cela nous étions d'avis. Mais laquelle ? Et à qui appartenait-il de tuer les bêtes sauvages ? [...] Les cerfs étaient-ils nos derniers dieux ? Avec les lynx, les ours et les loups ? Devaient-ils avoir le même statut qu'eux ? C'est-à-dire un autre statut que celui de viande ? Un autre que celui de trophée ? Le statut de frères ? Frère Loup ? Sœur Tique ? C'est-à-dire le statut d'une autre espèce nous rappelant que le monde n'est pas seulement humain ? (LS, p. 132)

Le texte met en place ici des éléments d'une casuistique, discipline qui depuis la charge de Pascal n'a pas bonne réputation en littérature mais dont il est difficile de se dispenser dans le cadre d'une réflexion sur l'éthique animale. Et La Survivance l'aborde avec une certaine dose d'ironie : l'allusion à " Sœur Tique » fait non seulement contrepoint au romantique « Frère Loup », elle fait résonner encore la méfiance envers le parasite exprimée ailleurs dans le roman. La tique est en effet, par la maladie de Lyme qu'elle véhicule, la menace la plus importante qui pèse sur les pionniers de la forêt: "Notre danger le plus sérieux, celui qui nous guettait vraiment, il était là, dans cette petite chose ronde, autonome, philosophique, explosive. Malgré Deleuze, on se méfiait d'elle » (LS, p. 118). L'ironie est à double détente puisque rappeler le prestige « intellectuel » qui se rattache à la tique en raison des commentaires que Deleuze lui a consacrés dans le prolongement de Jacob von Uexküll, est simultanément une manière de pointer le danger bien concret qu'elle représente dans la " vraie vie ».

Hunzinger rappelle qu'il importe de prendre conscience du fait que le monde n'est pas seulement humain, mais en même temps que l'on ne saurait se contenter de déplacer la frontière pour inclure les mammifères - ou certains d'entre eux - sur la base de leur capacité à souffrir ou à ressentir certaines émotions. Le problème se déplace en effet avec la nouvelle frontière, ou pour reprendre les termes du roman : la tique est-elle de ces animaux avec lesquels il convient de se comporter en frère et sœur?

Le roman met en place un univers dans lequel la place de l'homme est systématiquement pensée en relation avec le reste de la nature, animée mais aussi inanimée. La narratrice profite de l'opinion favorable que des visiteurs ont de leur « coin de paradis », pour amorcer une réflexion sur ce qu'il faut pour qu'un lieu soit complet : 


\section{DIRE LE LIEU POUR FAIRE NAÎTRE DES HISTOIRES}

Pourquoi, quand il y a seulement des herbes, des arbres, des rochers, n'est-ce pas le paradis ? Pourquoi, quand il y a seulement des humains, n'est-ce toujours pas le paradis? Pourquoi faut-il qu'il y ait des bêtes pour être au paradis? Peut-être parce que les dieux sont des animaux nietzschéens, indestructibles et heureux. Ce qui expliquerait pourquoi c'est seulement grâce à notre corps mortel, animal et heureux, qu'on devient éternel. (LS, p. 138)

La réflexion retrouve ici cet autre axe important du texte, déjà abordé, et qui, en contraste avec la jeunesse omniprésente dans Bambois, se déploie autour de la question du vieillissement et de la mort. Le paragraphe cité rappelle que la matérialité du monde demeure première ; comme l'infirmierphotographe, la narratrice se montre « à la fois sensible au destin des vieux et au silence des bêtes » $(L S$, p. 229).

\section{Moins, c'est plus : économie et écologie}

À vingt ans, Melu et Pagel regardaient vers l'avenir : même si le projet du jeune couple s'inscrit dans la quête d'un mode de vie alternatif, Bambois demeure un récit dans lequel l'accroissement de biens occupe une place importante. L'acquisition des terres, l'achat des brebis, les améliorations apportées aux fermes comme le commerce du tissage sont évoqués dans le détail. Hunzinger adopte volontiers le ton de la " victoire » $(B$, p. 157) et elle rappelle le " succès qui [leur] est tombé dessus " $(B$, p. 140), expliquant d'ailleurs l'arrivée toujours plus fréquente de visiteurs en quête d'un modèle à suivre. La Survivance met d'autres accents : n'ayant à leur âge plus rien à construire, Sils et de Jenny placent leur vie sous le signe de l'économie.

Initialement leur dépouillement s'explique par la faillite de la librairie, mais rapidement le mode de vie simple se trouve revendiqué comme un libre choix : «On conserve trop de choses » (LS, p. 26) explique Jenny qui imagine une simplicité qui rejoindrait celle de la montagne. La nécessité de se débarrasser du superflu se fait entendre telle une basse continue : « On va s'alléger » ( $L S$, p. 57$)$, répètera Sils avant le départ pour la forêt. Même quand les circonstances de vie deviendront extrêmement difficiles, le couple préfère envisager un dépouillement plus grand encore plutôt que d'accepter de se laisser déloger : " On se ferait une cabane moins grande et tout à fait cachée, à trois montagnes d'ici » (LS, p. 252).

Dans une recension du premier Bambois, Bernard Soulé avançait, observant la réussite du projet des Hunzinger, qu' « aujourd'hui, la "décivilisation" est possible, quoique pratiquement réservée à une minorité de privilégiés $»^{11}$. Mais La Survivance montre que de décivilisation, il n'est plus question en 2012 : il y aurait même plutôt surcivilisation, dont l'indice majeur serait la multiplication des références littéraires et artistiques, essentielles à l'architecture du roman. Mais cette surdétermination culturelle s'accompagne, comme l'illustre la place dévolue à l'idée d'économie, d'une adhésion au principe de décroissance. Le concept, né précisément au début des années 70, n'était pas encore central à cette époque largement productiviste mais il occupe une place importante dans des visions du monde alternatives qui se mettent en place en ce début de $\mathrm{XXI}^{\mathrm{e}}$ siècle.

Melu pouvait encore s'émouvoir à l'écoute du bruit des scies à chaîne : «De très loin dans les forêts, j'entends la tronçonneuse et c'est comme un appel, c'est une explosion animale, une agressivité sexuelle qui me fait frémir » $(B, \mathrm{p}$. 105). Jenny est sensible à bien autre chose, que le lieu où se situe leur nouvelle maison offre à profusion : «Tout est ouvert et donne sur le vide, le sillage du vide, l'appel de l'air, le cri des buses » (LS, p. 66).

Au-delà de l'endroit où se situe leur maison, la narratrice de La Survivance donne aussi forme aux lieux en faisant résonner les enjeux environnementaux contemporains. L'on est loin d'une écologie militante, mais une sensibilité s'exprime clairement à travers les choix des images. Les grands bourgeois ridicules - voire les «malades mentaux » $(B$, p. 160) - que les chasseurs étaient dans Bambois, se sont transformés en " parvenus analphabètes pour temps post-nucléaires » $(L S$, p. 228), conformément à un glissement de valeurs qui a vu le rappel de la responsabilité écologique prendre le pas sur celui des responsabilités sociales.

\footnotetext{
${ }^{11}$ SoulÉ Bernard, « Vivre en marge de la société », Le Figaro, 21 mars 1973.
} 
Une sensibilité proche s'exprime autour de la question de l'élevage industriel, abordée à l'occasion des villages traversés :

soudain de lourdes, de ténébreuses odeurs de porcherie nous barraient la route, nous poursuivaient longtemps. [Sils] bourdonnait à son habitude : Ce n'est pas très horrible, Jenny, c'est juste une sorte d'horreur, pas l'horreur des banlieues mais l'horreur agricole. (LS, p. 169)

La Survivance fait surgir les lieux en les situant dans le cadre de débats contemporains qui entourent la question du futur de la terre. Alors que Bambois, un des rares récits qui nous sont parvenus pour témoigner de ce qu'ont pu être les années du « retour à la terre », s'ancrait dans des lieux-dits bien réels en s'autorisant un regard bucolique, le roman de 2012 se saisit du lieu pour dire d'autres préoccupations. L'éventail des histoires s'élargit : inspirées par la sauvagerie essentielle de l'endroit, elles font résonner maintenant l'expérience sensorielle du monde, les recherches des artistes, la solitude des ermites, le partage avec les animaux, le vieillissement des corps et les interrogations concernant l'avenir de la terre.

Si pour Warburg « survivance » référait, comme le roman le rappelle, à « la capacité des images à revenir nous hanter ", le texte de La Survivance montre quant à lui la capacité de Hunzinger à faire vivre un lieu sans occulter la menace que l'homme fait peser sur ce dernier. Quand l'écrivaine lit le paysage à travers des réminiscences littéraires, il est révélateur qu'elle choisisse d'insister sur les enjeux environnementaux. L'imaginaire embraye sur la forme de certains nuages et transporte la narratrice sous des latitudes éloignées :

Et aussi qu'on se retrouve dans le Montana pas loin de Jim Harrison. Il arrivait encore que se forme dans le ciel une sorte de champignon d'un gris maléfique, boursouflé, délirant, là-bas du côté de Fessenheim. On se serait soudain crus en pleine catastrophe nucléaire. Et alors nous nous serions vite réfugiés dans la grotte de la source où nous aurions heureusement prévu de stocker des vivres pour trois mois, refermant l'entrée avec un bouchon de tourbe, et dans la plaine, déjà, ils auraient tous été morts, et de leurs corps, il ne serait resté que leurs ombres. ( $L S$, p. 81$)$

Par les associations que font naître les nuages, la nature sauvage de Harrisson se trouve juxtaposée au paysage de la centrale nucléaire de Fessenheim, située une cinquantaine de kilomètres au sud-est de La Survivance. La petite fable montrant Sils et Jenny dans la peau des survivants d'un accident nucléaire apparaît alors comme une miniature relocalisée du roman.

Au jeu du « on ferait comme si » l'île en montagne émerge maintenant au-dessus d'un paysage post-apocalyptique pour rappeler au lecteur la responsabilité qu'il a envers le futur de la planète.

\section{Bibliographie}

FINKEL Michael, The Stranger in the Woods, New York, Penguin Random House, 2017.

HUNZINGER Claudie, Bambois, la vie verte, Paris, Stock2, coll. « Vivre », 1973.

HunZINGER Claudie, La survivance, Paris, Grasset, 2012.

MARTIN Jean-Pierre, Sabots suédois, Paris, Fayard, 2004.

DE TON Delfeil, pseudonyme de RouSSEL Henri, «Bambois, la vie verte, bouquin de Cl. Hunzinger », Charlie Hebdo, n 124, lundi 2 avril 1973, p. 13.

Isabelle, « Bambois, La vie verte », Charlie Hebdo, n 129, lundi 9 mai 1973, sp.

SCHOENTJES Pierre, « Solitaires dans la nature : "L'art est ce qui rend la vie plus intéressante que l'art" », Les Temps modernes, $\mathrm{n}^{\circ}$ 695, 2017, p. 141-166.

SCHOENTJEs Pierre, Ce qui a lieu, Essai d'écopoétique, Marseille Wildproject, 2015.

SOUDET Pierre, «Les moutons des Vosges », L'Express, 21-27 mai 1973, p. 157. 


\section{DIRE LE LIEU POUR FAIRE NAÎTRE DES HISTOIRES}

SOULÉ Bernard, « Vivre en marge de la société », Le Figaro, 21 mars 1973. 